\title{
JAK2/PD-L1/PD-L2 (9p24.1) amplifications in renal cell carcinomas with sarcomatoid transformation: implications for clinical management
}

\author{
Sounak Gupta ${ }^{1}$ John C. Cheville ${ }^{2} \cdot$ Achim A. Jungbluth $^{1} \cdot$ Yanming Zhang $^{1} \cdot$ Lei Zhang $^{1} \cdot$ Ying-Bei Chen $\mathbb{D}^{1}$. \\ Satish K. Tickoo ${ }^{1} \cdot$ Samson W. Fine ${ }^{1} \cdot$ Anuradha Gopalan $^{1} \cdot$ Hikmat A. Al-Ahmadie $^{1} \cdot$ Sahussapont J. Sirintrapun ${ }^{1}$. \\ Kyle A. Blum ${ }^{3,4} \cdot$ Christine M. Lohse $^{5}$ - A. Ari Hakimi ${ }^{3,4} \cdot$ R. Houston Thompson ${ }^{6} \cdot$ Bradley C. Leibovich $^{6}$. \\ Michael F. Berger ${ }^{1} \cdot$ Maria E. Arcila ${ }^{1} \cdot$ Dara S. Ross $^{1} \cdot$ Marc Ladanyi $^{1} \cdot$ Cristina R. Antonescu $^{1} \cdot$ Victor E. Reuter $^{1}$
}

Received: 5 December 2018 / Revised: 14 February 2019 / Accepted: 6 March 2019 / Published online: 17 April 2019

(c) United States \& Canadian Academy of Pathology 2019

\begin{abstract}
Amplifications of $J A K 2, P D-L 1$, and $P D-L 2$ at 9p24.1 lead to constitutive expression of PD-L1. This, coupled with JAK2activation dependent upregulation of $P D-L 1$ and adaptive/induced expression leads to higher tumor PD-L1 expression and immune evasion. Renal tumors were therefore evaluated for 9p24.1 amplifications. A combination of next generation sequencing-based copy number analysis, fluorescence in situ hybridization for JAK2/INSL6 and PD-L1/PD-L2 and immunohistochemistry for phospho-STAT3 (downstream target of JAK2), PD-L1, PD-L2, and PD-1 was performed. In this study we interrogated a "Discovery" cohort of 593 renal tumors, a "Validation" cohort of 398 high-grade renal tumors, The Cancer Genome Atlas (879 cases) and other public datasets (846 cases). 9p24.1 amplifications were significantly enriched in renal tumors with sarcomatoid transformation $(5.95 \%, 15 / 252)$ when compared to all histologic subtypes in the combined "Discovery", "Validation" and public datasets $(16 / 2636,0.6 \%, p<0.00001)$. Specifically, 9p24.1 amplifications amongst sarcomatoid tumors in public datasets, the "Discovery" and "Validation" cohorts were 7.7\% (6/92), 15.1\% (5/33), and 3.1\% (4/127), respectively. Herein, we describe 13 cases and amplification status for these was characterized using next generation sequencing $(n=9)$ and/or fluorescence in situ hybridization $(n=10)$. Correlation with PD-L1 immunohistochemistry $(n=$ 10) revealed constitutive expression (mean H-score: $222 / 300, n=10$ ). Analysis of outcomes based on PD-L1 expression in tumor cells performed on 282 cases ("Validation" cohort) did not reveal a significant prognostic effect and was likely reflective of advanced disease. A high incidence of constitutive PD-L1 expression in tumor cells in the "Validation" cohort (H-Score $\geq 250 / 300$ ) was noted amongst 83 rhabdoid (6\%) and 127 sarcomatoid renal tumors (7.1\%). This suggests additional mechanisms of constitutive expression other than amplification events. Importantly, two patients with 9p24.1amplified sarcomatoid renal tumors showed significant response to immunotherapy. In summary, a subset of renal tumors with sarcomatoid transformation exhibits constitutive PD-L1 overexpression and these patients should be evaluated for enhanced response to immunotherapy.
\end{abstract}

Supplementary information The online version of this article (https:// doi.org/10.1038/s41379-019-0269-x) contains supplementary material, which is available to authorized users.

\section{Victor E. Reuter}

reuterv@mskcc.org

1 Department of Pathology, Memorial Sloan Kettering Cancer Center, New York, NY, USA

2 Department of Laboratory Medicine and Pathology, Mayo Clinic, Rochester, MN, USA

\section{Introduction}

Janus kinase 2 (JAK2) is one of four JAK-domain containing tyrosine kinases, which promotes downstream

3 Department of Surgery, Urology Service, Memorial Sloan Kettering Cancer Center, New York, NY, USA

4 Department of Human Oncology and Pathogenesis Program, Memorial Sloan Kettering Cancer Center, New York, NY, USA

5 Department of Health Sciences Research, Mayo Clinic, Rochester, MN, USA

6 Department of Urology, Mayo Clinic, Rochester, MN, USA 
oncogenic signaling events through the signal transducer and activator of transcription (STAT) proteins [1]. Diverse mechanisms of $J A K 2$ activation commonly seen in myeloproliferative neoplasms include activating mutations (JAK2 p.V617F, exon12 mutations) and signaling through mutant thrombopoietin receptors (MPL p.W515L/K) [2]. Another mechanism of $J A K 2$ activation which is being increasingly recognized in both hematologic malignancies as well as in solid tumors involves genomic amplification events [3, 4]. Amongst hematologic malignancies, these events have been described in classical Hodgkin lymphoma and mediastinal large B-cell lymphoma, while the expanding list of solid tumors with such alterations includes EBV-positive gastric adenocarcinoma, oral cavity squamous cell, lung small cell and breast carcinomas [4-12]. A recent pan-cancer study of 118,187 tumors revealed this alteration to be much more ubiquitous, as it was observed in over 100 different tumor types, with an overall frequency of $0.7 \%$ [3]. Interestingly, amongst solid tumor types where greater than 40 cases were evaluated, the highest prevalence of this alteration was reported in renal cell carcinomas with sarcomatoid change (6.1\%, 4 of 66 cases) [3].

9p24.1 amplifications have significant clinical implications for immunotherapy in both hematologic neoplasms as well as in solid tumors [3, 12-17]. Genes present at this genomic locus include $J A K 2$, programmed death ligand-1 (PD-L1, also known as CD274/ PDCD1LG1) and programmed death ligand-2 (PD-L2, also known as CD273/ $P D C D 1 L G 2)[3,4]$. As increased JAK2 signaling has been shown to lead to secondary overexpression of PD-L1 on the tumor cell surface, this 9p24.1 amplification event has a synergistic effect leading to constitutive over-expression of PD-L1 [4]. In support of this thesis, we identified a unique biologic phenomenon in a lung adenocarcinoma which harbored a 1.5-fold germline intragenic gain of exon16 to 25 of $J A K 2$, which codes for the C-terminus including the kinase domain (Supplementary Fig. 1a). The corresponding biopsy specimen of the lung adenocarcinoma (Supplementary Fig. 1b-e) demonstrated diffuse, strong nuclear expression of phospho-STAT3, which is a surrogate marker of activated $J A K 2$-signaling [1]. In addition, strong membranous expression of PD-L1 was noted in over $90 \%$ of neoplastic cells in this biopsy specimen, supporting JAK2dependent signaling as having an important role in PD-L1 upregulation.

PD-L1/PD-L2 expressed on the tumor cell surface help evade the T-cell mediated immune response within the tumor microenvironment by binding the programmed death 1 (PD-1) receptor on these cells [18]. Immune checkpoint inhibitors (anti-PD-1 agents: Nivolumab, Pembrolizumab; anti-CTLA-4 agents: Ipilimumab; anti-PD-L1 agents: Atezolizumab, Avelumab) restore this immune response and their use is gaining popularity as a therapeutic modality for renal cell carcinomas [19-25]. Based on current National Comprehensive Cancer Network guidelines (NCCN Clinical Practice Guidelines in Oncology, NCCN Guidelines ${ }^{\circledR}$, Kidney Cancer V.2.2018) the use of nivolumab is indicated for the treatment of patients with advanced renal cell carcinomas who have received prior anti-angiogenic therapy. Specifically, it is a category 1 recommendation (uniform consensus based on high-level evidence) for subsequent therapy of advanced clear cell renal cell carcinoma and a category $2 \mathrm{~A}$ recommendation (uniform consensus based on lower-level evidence) for management of advanced non-clear cell renal cell carcinoma patients. Currently at least nine clinical trials are actively exploring the role of immunotherapy in the management of advanced renal cell carcinomas (https://clinicaltrials.gov; Nivolumab: NCT01668784; Nivolumab \& Ipilimumab: NCT02231749; Pembrolizumab: NCT02853344, NCT02853331, NCT02212730, and NCT02432963; Atezolizumab: NCT02420821, NCT01984242; Avelumab: NCT01772004).

Given the growing body of literature documenting favorable response to immunotherapy for 9p24.1 amplified tumors [3,13-17], in this study we have sought to determine the prevalence of these events in renal cell carcinomas and to characterize relevant clinicopathologic features of these tumors. These alterations were found to be enriched in renal cell carcinomas with sarcomatoid transformation. According to the WHO classification, sarcomatoid renal cell carcinoma is not a specific tumor entity and such transformation can be observed in different histological subtypes.

\section{Materials and methods}

\section{Patient specimens}

This study was approved by the institutional review board and involved analysis of molecular profiling data for all renal tumors profiled by a next generation sequencing (NGS)-based assay, Memorial Sloan Kettering Cancer Center Integrated Mutation Profiling of Actionable Cancer Targets (MSK-IMPACT), as part of an institutional clinical cancer genomics initiative [26-28]. This included 307 clear cell renal cell carcinomas, 74 unclassified renal cell carcinomas, 54 papillary renal cell carcinomas, 42 renal cell carcinomas not otherwise specified, 30 chromophobe renal cell carcinomas, 26 translocation associated renal cell carcinomas, 10 fumarate hydratase deficient renal cell carcinomas, 13 renal cell carcinomas with sarcomatoid transformation, 8 renal medullary carcinomas, 4 mucinous tubular and spindle cell carcinomas, 3 clear cell papillary renal cell carcinomas, and 2 collecting duct carcinomas. 
Whole slide sections from an additional 20 cases of sarcomatoid renal cell carcinoma were analyzed using fluorescence in situ hybridization for copy number alterations at chromosome 9p24.1. These cases comprised the "Discovery cohort" for identification of 9p24.1 amplified tumors (593 renal tumors, including 33 cases of sarcomatoid renal cell carcinoma).

Following institutional review board approval, the Mayo Clinic Nephrectomy Registry was queried to identify 398 patients treated with radical or partial nephrectomy for sporadic, unilateral WHO/ISUP grade 4 renal cell carcinomas (based on current WHO/ISUP criteria) for cases diagnosed between 1980 and 2016. These cases comprised the "Validation cohort" and 282 of these patients met inclusion criteria for further statistical analysis related to outcomes and PD-L1 expression status, even though only a small subset received immunotherapy. See Supplementary methods and Supplementary Table 1 for specific details.

\section{Immunohistochemistry and fluorescence in situ hybridization}

Immunohistochemistry was performed for PD1 (clone NAT105, prediluted, CELL MARQUE, Rocklin, CA), PDL1, PD-L2, phospho-STAT3 (PD-L1: clone E1L3N, 1:400 dilution, PD-L2: clone D7U8C, 1:20 dilution and phosphoSTAT3: clone M9C6, 1:250 dilution; Cell Signaling Technology, Danvers, MA) and TTF1 (clone 8G7G3/1, prediluted, Ventana, Tucson, Arizona). Immunohistochemistry with the PD-L1 antibody was clinically validated internally against the PD-L1 22C3 clone from Dako and found to be comparable. Fluorescence in situ hybridization for JAK2/INSL6 and PD-L1/PD-L2 genes was performed as previously described $[29,30]$. See supplementary methods for additional details.

\section{Next generation sequencing-based copy number assessment: 9p24.1 amplifications}

Details of the MSK-IMPACT assay have been previously reported [26-28, 31]. Based on previously reported criteria, amplifications were defined as a fold change $\geq 2.0$, while copy number gains/borderline amplifications were defined as a fold change $\geq 1.5$ but $<2$ [31]. See supplementary methods for details.

\section{Literature review and data extraction from the cancer genome atlas datasets}

The publicly available cBioPortal.32e34 platform was used to analyze data from The Cancer Genome Atlas and other public datasets [32]. See supplementary methods for details.

\section{Statistical analysis}

Associations of PD-L1 H-score and patient outcomes were assessed using the Validation cohort from the Mayo Clinic Nephrectomy Registry (282 cases). Cancer-specific and distant metastases-free survival were estimated using the Kaplan-Meier method with the duration of follow-up calculated from the date of nephrectomy to the date of distant metastases, death, or last follow-up. Associations with time to death from renal cell carcinoma and time to distant metastases were evaluated using Cox proportional hazards regression models and summarized with hazard ratios and 95\% confidence intervals. All models were adjusted for year of nephrectomy to account for changes in clinical and pathologic features over time. Statistical analyses were performed using SAS version 9.4 (SAS Institute, Cary, NC) and $\mathrm{R}$ version 3.4.2 (R Foundation for Statistical Computing, Vienna, Austria). All tests were two-sided and $p$-values $<0.05$ were considered statistically significant. See supplementary methods for details.

\section{Results}

\section{Assessment of 9p24.1 amplifications}

Genes located at the 9p24.1 locus include JAK2, INSL6, $P D-L 1$, and $P D-L 2$ (Fig. 1a). Molecular profiling of copy number alterations using $J A K 2, P D-L 1$, and $P D-L 2$ exonic probes successfully identified these alterations. For instance, profiling of an area of sarcomatoid transformation in a chromophobe renal cell carcinoma revealed a 3.5 -fold genomic amplification at this locus (Fig. 1b, case 1).

These alterations were confirmed by a combination of fluorescence in situ hybridization and immunohistochemistry. A representative case with a 2.7-fold 9p24.1 amplification detected by MSK-IMPACT, in an area of sarcomatoid transformation in a clear cell renal cell carcinoma, was confirmed using fluorescence in situ hybridization for $P D-L 1 / P D-L 2$ (Fig. 2a, b, Case 4), and immunohistochemistry for PD-L1 (Fig. 2c, Case 4) and PDL2 (not shown). Similarly, fluorescence in situ hybridization for JAK2/INSL6 (Fig. 2d, e, Case 1) and corresponding immunohistochemistry for phospho-STAT3 (Fig. 2f, Case 1), in an area of sarcomatoid transformation in a chromophobe renal cell carcinoma, were supportive of a 3.5-fold genomic amplification at 9p24.1.

\section{Pattern of PD-L1 Expression by Immunohistochemistry}

PD-L1 protein expression has been commonly described to occur in an adaptive and constitutive pattern [18]. 
Fig. 1 Copy number assessment (Case 1). A schematic representation of genes present at the 9p24.1 locus is shown (a not to scale). A copy number plot for a sarcomatoid renal cell carcinoma profiled using MSKIMPACT has been depicted (b). Relative ( $\log 2)$ tumor/normal ratios (y-axis) and corresponding chromosomes ( $x$-axis) are displayed, with each blue dot representing an individual probe region. Amplified regions are shown in red and show a 3.5fold amplification for $J A K 2 / P D$ $L 1$ and $P D-L 2$ at $9 \mathrm{p} 24.1$. MSKIMPACT, Memorial Sloan Kettering Cancer Center Integrated Mutation Profiling of Actionable Cancer Targets
A
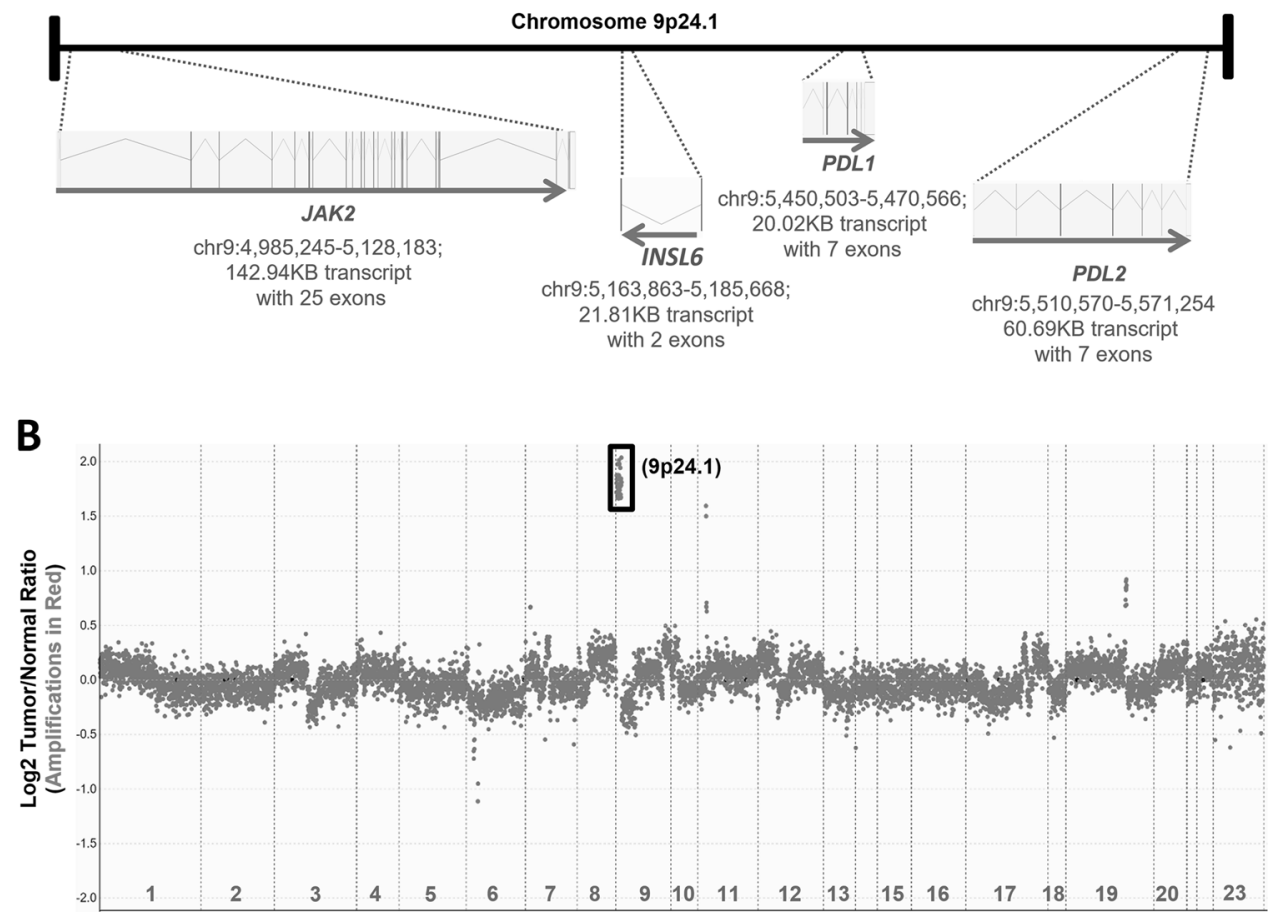

Constitutive expression is characterized by diffuse membranous expression in tumor cells independent of their association with adjacent activated T-lymphocytic infiltrates within the tumor microenvironment (Supplementary Fig. 2a-f) and this pattern was observed in all renal cell carcinomas that were identified to have 9p24.1 amplifications. The adjacent non-neoplastic renal parenchyma (Supplementary Fig. 2a, b), tumor-infiltrating lymphocytes (Supplementary Fig. 2c, d) and stromal elements (Supplementary Fig. 2e, f) lacked PD-L1 expression. On the other hand, adaptive expression in tumor cells was found to be geographically associated with tumor-infiltrating lymphocytes (Supplementary Fig. 3a-d). This pattern was commonly seen in renal cell carcinomas that lacked 9p24.1 amplifications. Of note, a few 9p24.1 amplified tumors demonstrated a combined pattern of PD-L1 protein expression that included an adaptive pattern of expression adjacent to a cuff of tumor-infiltrating lymphocytes at the tumor front, in addition to a constitutive pattern of expression seen in the rest of the tumor (Supplementary Fig. 4a-d). Furthermore, in these cases tumor-infiltrating lymphocytes exhibited a robust expression of PD1 (Supplementary Fig. 4e, f).

\section{Prevalence of 9p24.1 amplifications in renal cell carcinomas}

Interrogation of the "Discovery" cohort of 593 renal tumors led to the identification of 5 cases with 9p24.1 amplifications, suggesting a prevalence of $0.8 \%$ (Table 1). Amongst 33 cases with sarcomatoid change in this cohort, the prevalence was $15.1 \%$ (5 cases). As this signature was only identified in high-grade renal tumors with sarcomatoid transformation, these cases were unlikely to have been specifically profiled in datasets looking at common histologic subtypes of renal cell carcinomas. For instance, analysis of The Cancer Genome Atlas datasets pertaining to 879 renal tumors, led to the identification of three 9p24.1-amplified tumors (Table 1) [33-36]. All three cases were clear cell renal cell carcinomas, suggesting a prevalence of $0.6 \%$ (3 of 533 cases) in this cohort and two of these three tumors exhibited sarcomatoid transformation or rhabdoid features (information on the third tumor was not available). Analysis of publicly available datasets from a study of metastatic chromophobe renal cell carcinomas led to the identification of two (of 60, 3.3\%) additional cases (Table 1) [37]. Of note, both cases exhibited sarcomatoid transformation.

A review of the literature in this regard revealed two additional informative studies. The first study reported results from molecular profiling of a cohort of sarcomatoid renal tumors and identified 2 of 26 cases, suggesting a prevalence of $7.7 \%$ (Table 1) [38]. Specifically, an amplification of $J A K 2$ was identified in the sarcomatoid component of two renal tumors, at least one of which had a lower grade clear cell component where this signature was absent [38]. However, the authors of this study did not interrogate other genes present at the 9p24.1 locus. A second pancancer study that analyzed 118,187 tumors for $9 \mathrm{p} 24.1$ 
Fig. 2 Histopathology, fluorescence in situ hybridization and immunohistochemistry (Case 1 \& 4). Representative H\&Estained images of $9 \mathrm{p} 24.1$ amplified sarcomatoid renal cell carcinomas (a Case 4, 2.7-fold amplification by MSK-

IMPACT, $\times 400$ magnification; $d$ Case 1, 3.5-fold amplification by MSK-IMPACT, $\times 200$ magnification), corresponding fluorescence in situ hybridization (b Red: $P D-L 1$, Green: $P D-L 2$, Aqua:

Centromere; e Orange: $J A K 2$, Green: INSL6, Red: Centromere) and immunohistochemistry (c PD-L1, x400 magnification; $f$ phospho-STAT3, $\times 200$ magnification) have been depicted. MSK-IMPACT, Memorial Sloan Kettering Cancer Center Integrated

Mutation Profiling of Actionable Cancer Targets
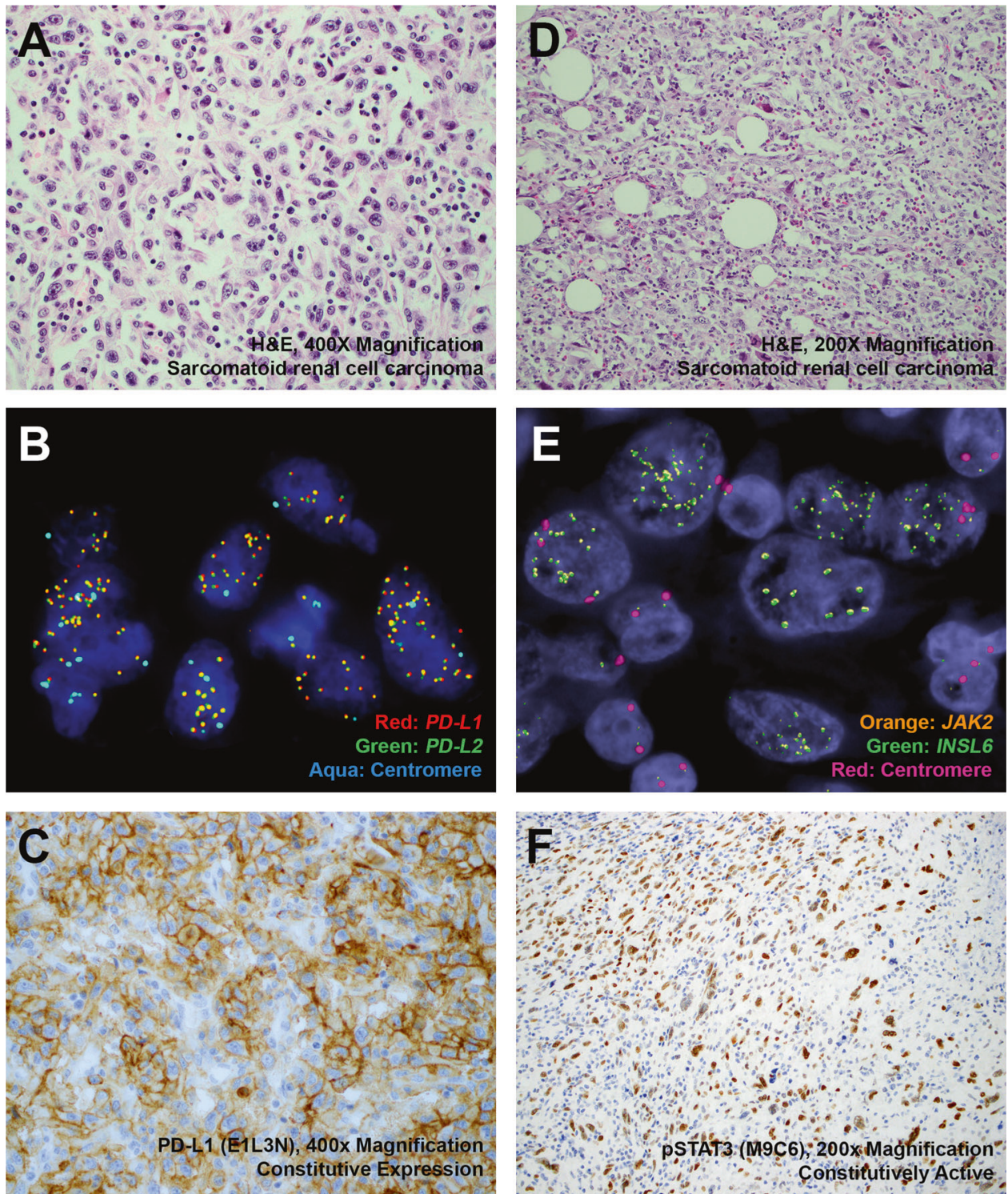

amplifications, reported the highest prevalence of this alteration in sarcomatoid renal tumors (4 of 66 cases, $6.1 \%$, Table 1) amongst solid tumor types where greater than 40 cases had been profiled [3]. However, no clinicopathologic features were reported for these cases [3].

In summary, while sarcomatoid renal tumors profiled as part of the "Discovery" cohort revealed a prevalence of $15.1 \%$ ( 5 of 33 cases), other published studies that focused specifically on the same histology revealed a prevalence of $6.1 \%$ ( 4 of 66 cases) to $7.7 \%$ ( 2 of 26 cases) [3, 38]. To address the prevalence of this alteration further, tissue microarrays with representative tumor from 398 high-grade renal tumors were systematically screened for 9p24.1 amplifications using fluorescence in situ hybridization for JAK2/INSL6, as part of the "Validation" cohort. Confirmatory testing of positive cases was performed using the same technique on whole slides sections. Four cases identified using this approach had sarcomatoid transformation (of a total of 127 tumors with sarcomatoid features) suggesting a prevalence of $3.1 \%$ (Table 1). After combining the prevalence from "Discovery" and "Validation" cohorts, along with results from Malouf et al and Goodman et al, the overall prevalence of $9 \mathrm{p} 24.1$ amplifications in sarcomatoid renal tumors is $5.95 \%$ ( 15 of 252 cases) [3, 38]. This is significantly higher compared to all histologic subtypes in the combined "Discovery", "Validation", The Cancer Genome Atlas datasets and results reported by Goodman et al. $(16 / 2636,0.6 \%, p<0.00001)[3]$.

\section{9p24.1-amplified renal cell carcinoma: clinicopathologic features}

The clinicopathologic characteristics of 13 renal tumors with 9p24.1 amplifications ("Discovery" cohort: 5 cases; 


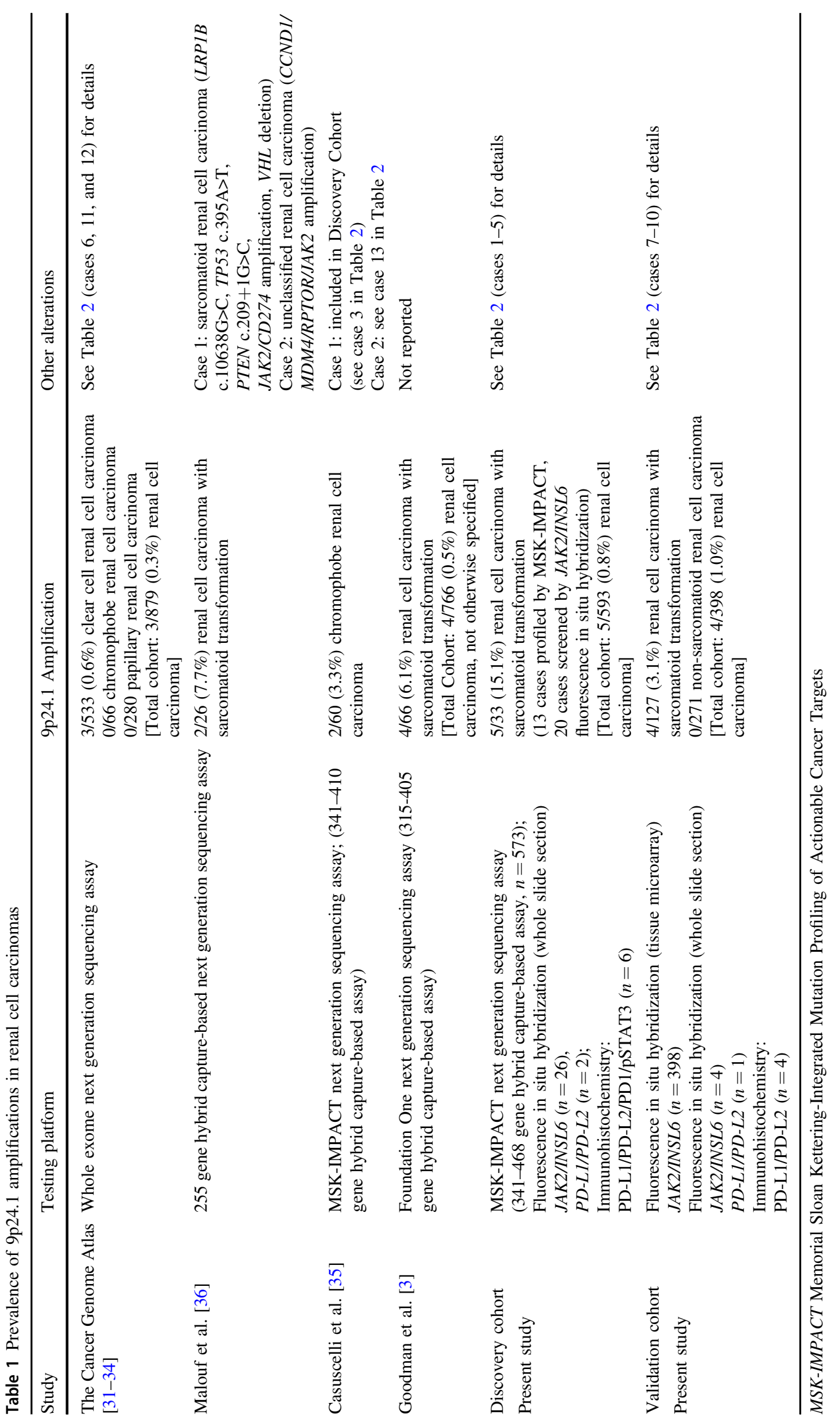


"Validation" cohort: 4 cases; TCGA: 3 cases; Casuscelli et al.: 1 case) are listed in Table 2 . The average age at nephrectomy for these patients was 62.8 years (range, 3184 years) and these tumors were predominantly identified in male patients ( 9 of 13,69\%). The mean tumor size was $11.3 \mathrm{~cm}$ (range, $6.3-20 \mathrm{~cm}$ ) based on what was reported for 11 (of 13) cases. Most of the cases were clear cell renal cell carcinomas ( 9 of $13,69 \%$ ) and 8 of these cases were WHO/ ISUP grade 4 with sarcomatoid or rhabdoid features. Of the remaining 4 cases, 3 were chromophobe renal cell carcinomas and two of these tumors exhibited sarcomatoid transformation. The fourth was a metastatic RCC, not otherwise specified, with extensive sarcomatoid transformation. In all, 11 of $13(84.6 \%)$ cases exhibited sarcomatoid transformation or rhabdoid features. In addition, 10 (of 12, $83 \%$ ) primary tumors were stage pT3 or higher.

Eight (of 13,62\%) patients died of disease at a mean follow-up of 11 months (range, 2-26 months). Of the two patients with organ confined tumors, one patient with pT2aNOM0 disease died of other causes at 181 months of follow up, while a second patient with pT1N0M0 disease was alive without disease at 27 months of follow up.

Of note, two patients with pT4 clear cell RCCs with sarcomatoid transformation were treated with immunotherapy. The first patient, Case 4 , received pembrolizumab as part of second line therapy. Pre-treatment imaging for this patient demonstrated widespread metastatic disease including involvement of the adrenal, muscle, bone as well as thoracic lymphadenopathy and involvement of both the right inferior pulmonary vein and left superior gluteal vein. Initial imaging studies showed stable disease, however, pembrolizumab was eventually discontinued after 16 months due to immune nephritis. This patient is alive with disease at 27.7 months of follow up. The second patient, Case 5, received atezolizumab as part of first line therapy. Imaging studies prior to the initiation of immunotherapy documented widespread metastatic disease in this case, as well. This included pulmonary, pleural, hepatic, osseous metastases as well as thoracic adenopathy and involvement of the nephrectomy bed. Follow up imaging at 3 months showed dramatic response particularly with decrease in the size and number of pulmonary metastasis, adenopathy, and nephrectomy bed involvement. At the time of discontinuation of immunotherapy at 14 months, this patient had radiologic evidence of stable disease. This patient subsequently had disease progression and died of disease at 26 months of follow up.

\section{9p24.1-amplified renal cell carcinoma: molecular profiling}

The 5 cases identified using MSK-IMPACT showed a 1.93.5-fold gain of genes at 9p24.1 (Table 3). These amplifications were confirmed using fluorescence in situ hybridization in all 5 cases as well as in 4 cases in the "Validation" cohort (Table 3). Interestingly, in a limited number of cases where a lower grade component was present, amplification was detected only in the higher-grade area with rhabdoid features or sarcomatoid transformation. A representative case has been illustrated in Fig. 3a-f (Case 4). Targeted/whole exome sequencing was used for an additional case from the cohort characterized by Casuscelli et al. and the 3 cases identified from The Cancer Genome Atlas datasets, respectively (Table 3) [37]. Of note, a single 9p24.1-amplified tumor in The Cancer Genome Atlas dataset (Case 6), had documented JAK2/PD-L1/PD$L 2$ mRNA expression $\geq 99^{\text {th }}$ percentile for the 510 -clear cell renal cell carcinomas profiled (Table 3) [36]. Furthermore, this case had structural rearrangements involving both JAK2 and PD-L1 (DOCK8-JAK2, CD274-KANK1, Table 4). The functional significance of these structural rearrangements in the background of an amplification at the 9p24.1 locus is uncertain [39, 40].

Corresponding immunostaining was performed for phospho-STAT3, PD-L1, PD1, and PD-L2 (Supplementary Fig. 5). Immunostaining for PD-L2 $(n=10$, mean H-score: 26.5) and phospho-STAT3 $(n=6$, mean H-score: 86.7) showed a poorer correlation with 9 p24.1 amplification status compared to PD-L1 expression $(n=10$, mean H-score: 222; Table 3).

Nine 9p24.1-amplified cases were profiled for mutational status and 3 of these cases revealed alterations involving either TP53/PTEN/RELN genes which have been reported to be associated with sarcomatoid transformation and poor outcomes (Table 4) [41]. Similarly, SETD2/BAP1/PBRM1 alterations have been reported to be associated with different rates of progression to sarcomatoid transformation. Of the 6-clear cell renal cell carcinomas profiled, only 1 case each had a mutation involving either SETD2, BAP1, or PBRM1 (Table 4) [41]. Recent studies have correlated the efficacy of immunotherapy with a high tumor mutation burden [42]. The mean tumor mutation burden for 5 cases profiled by MSK-IMPACT was 4 mutations/Megabase (range, 2-7.9) which was similar to all renal tumors within the institutional clinical sequencing cohort (3.0 mutations/ Megabase), suggesting limited utility of this metric in these tumors.

\section{PD-L1 expression status of high-grade renal cell carcinomas: prognostic significance}

Tissue microarrays with representative tumors from 398 high-grade renal tumors were evaluated for PD-L1 expression in tumor cells using immunohistochemistry (Fig. 4a). As expected, 9p24.1-amplified tumors showed significantly higher $\mathrm{H}$-scores (median: 242.5; interquartile range: 190- 


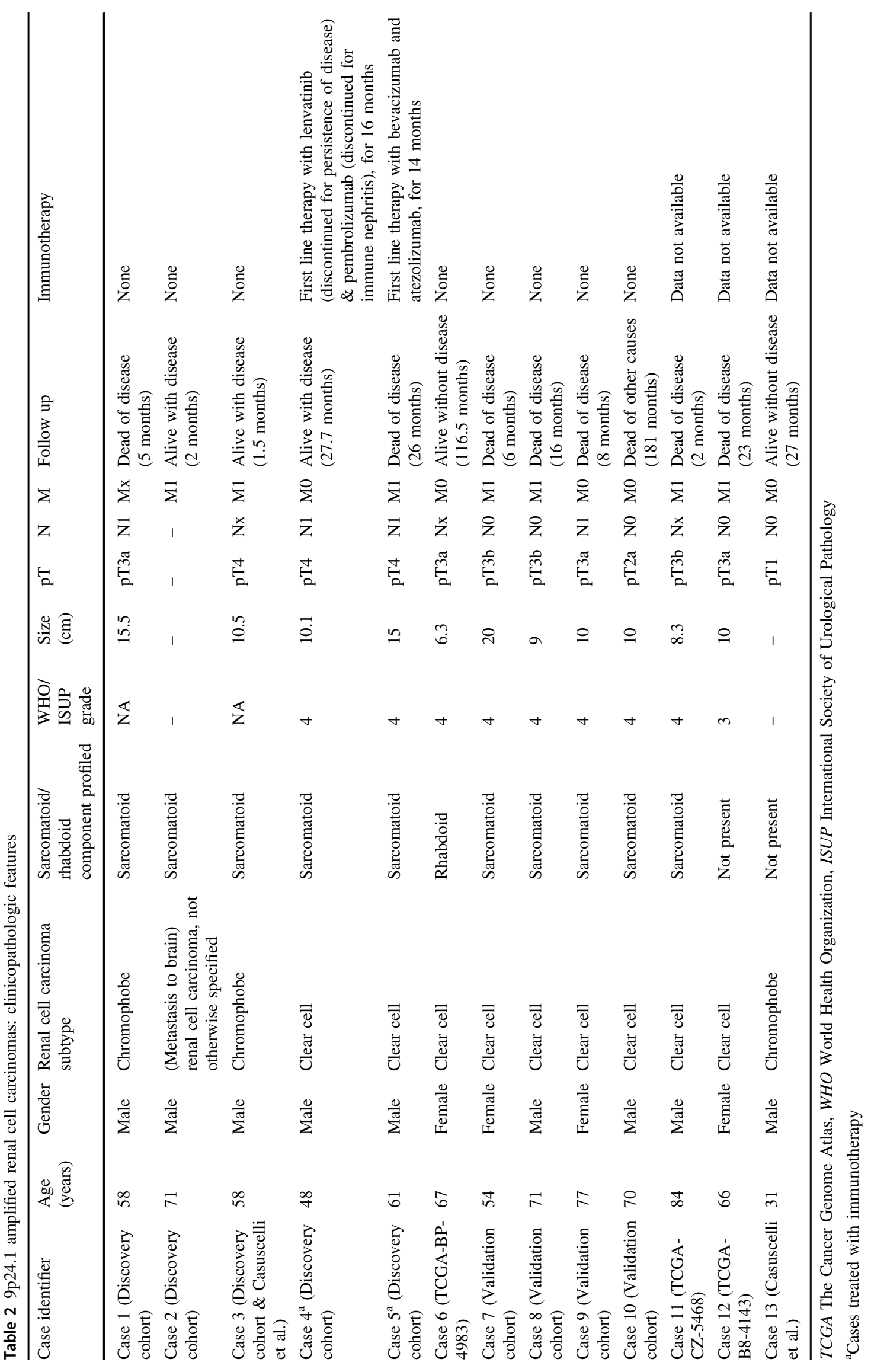




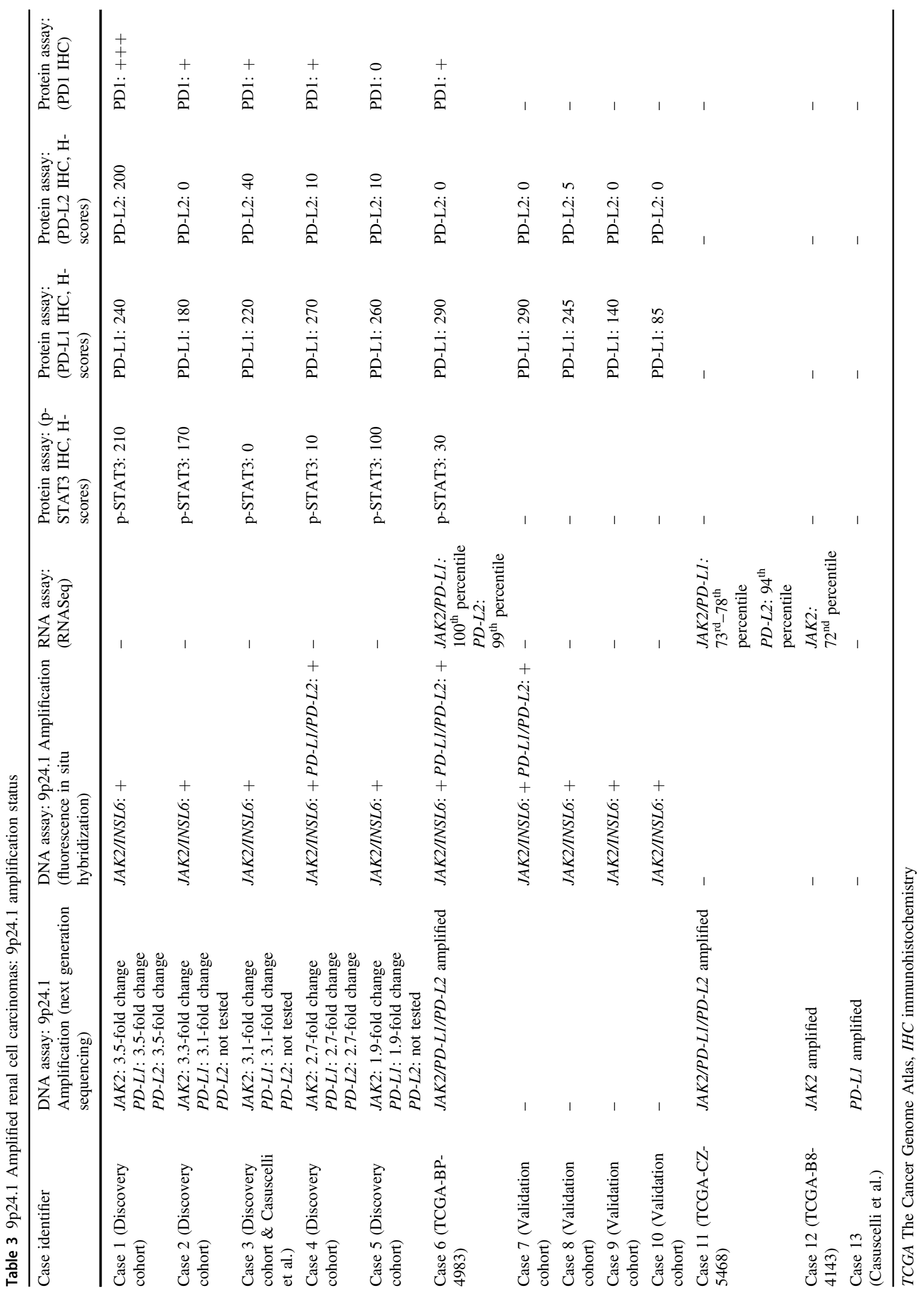


Fig. 3 Histopathology and Immunohistochemistry (Case 4). Representative H\&E stained images of a 9p24.1-amplified clear cell renal cell carcinoma with sarcomatoid transformation is depicted (a adjacent clear cell and sarcomatoid areas, $\times 40$ magnification; c representative area with clear cell morphology, $\times 200$ magnification; $\mathbf{e}$ representative area with sarcomatoid morphology, $\times 200$ magnification). Corresponding immunostaining for PD-L1 shows absence of expression in areas with a lower grade clear cell component (b left, $\times 40$ magnification; $\mathbf{d} \times 200$ magnification) and constitutive expression in areas with a higher grade sarcomatoid component (b right, $\times 40$ magnification; $\mathbf{f} \times 200$ magnification)
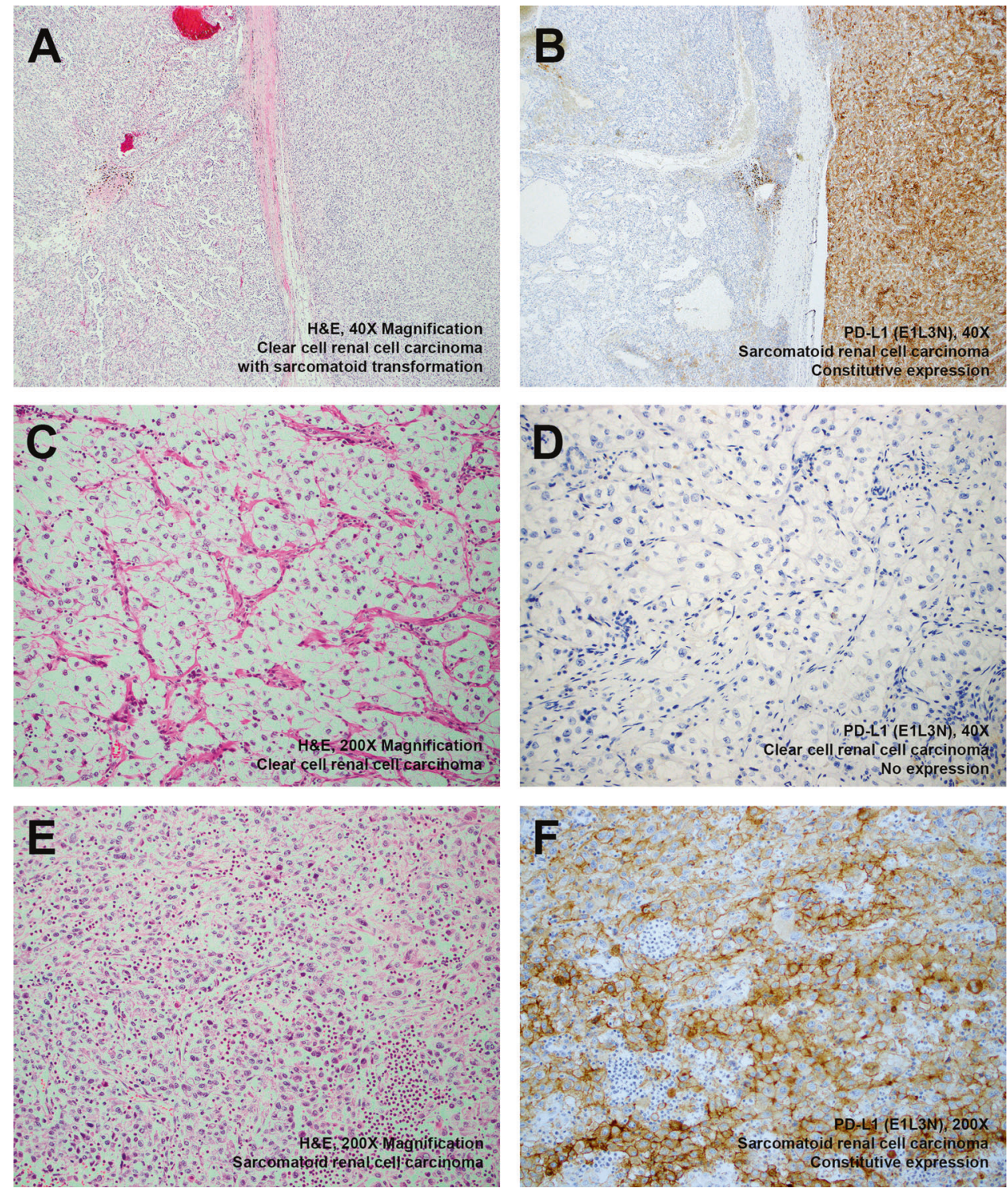

267.5, $n=10$ ) compared to non-9p24.1 amplified renal tumors. Similar to the results reported by Kawakami et al., sarcomatoid renal cell carcinomas showed significantly higher $H$-scores as well, with $\sim 27.6 \%$ of cases showing an $H$-score $\geq 50$ (Fig. 4b) [43].

Immunostaining for PD-L1 revealed significant heterogeneity, particularly when higher grade rhabdoid or sarcomatoid elements were present in a background of lowergrade renal cell carcinoma (Supplementary Fig. 6a-f). When estimating the $H$-score for such cases, an average score was assigned for all neoplastic cells irrespective of heterogeneity. On stratification of cases based on arbitrary $H$-score cutoffs ( $\geq 50, \geq 100, \geq 150, \geq 200$, and $\geq 250$ ), $6.0 \%$ (5 of 83 cases) of renal carcinomas with rhabdoid features and $7.1 \%$ ( 9 of 127 cases) of sarcomatoid renal tumors had a $\mathrm{H}$ score $\geq 250$ (Fig. 4b). This high level of PD-L1 expression ( $H$-score $\geq 250)$ suggests that many of these tumors might potentially have constitutive expression secondary to mechanisms other than 9p24.1 amplifications. This is supported by mRNA expression profiling data from The Cancer Genome Atlas datasets where 9\% (45 of 510) of clear cell, $11 \%$ (31 of 274) of papillary, and 11\% (7 of 65) of chromophobe renal cell carcinomas show increased mRNA expression of $J A K 2, P D-L 1$, and $P D-L 2$ [36].

PD-L1 $H$-scores for 282 patients in the "Validation" cohort were analyzed for cancer-specific survival and 176 of these patients who were M0 at nephrectomy were analyzed for distant metastases-free survival. Since nearly half of the tumors studied had PD-L1 H-scores of 0 , this feature was analyzed as continuous and after categorizing as 0 and $>0$. Cancer-specific survival rates $(95 \%$ confidence interval; number still at risk) at 1,2, and 3 years following surgery were $66 \%$ (60-72; 167), 49\% (44-6; 123), and 40\% (34-47; 88 ), respectively (Fig. 3c). The univariable association of PD- 


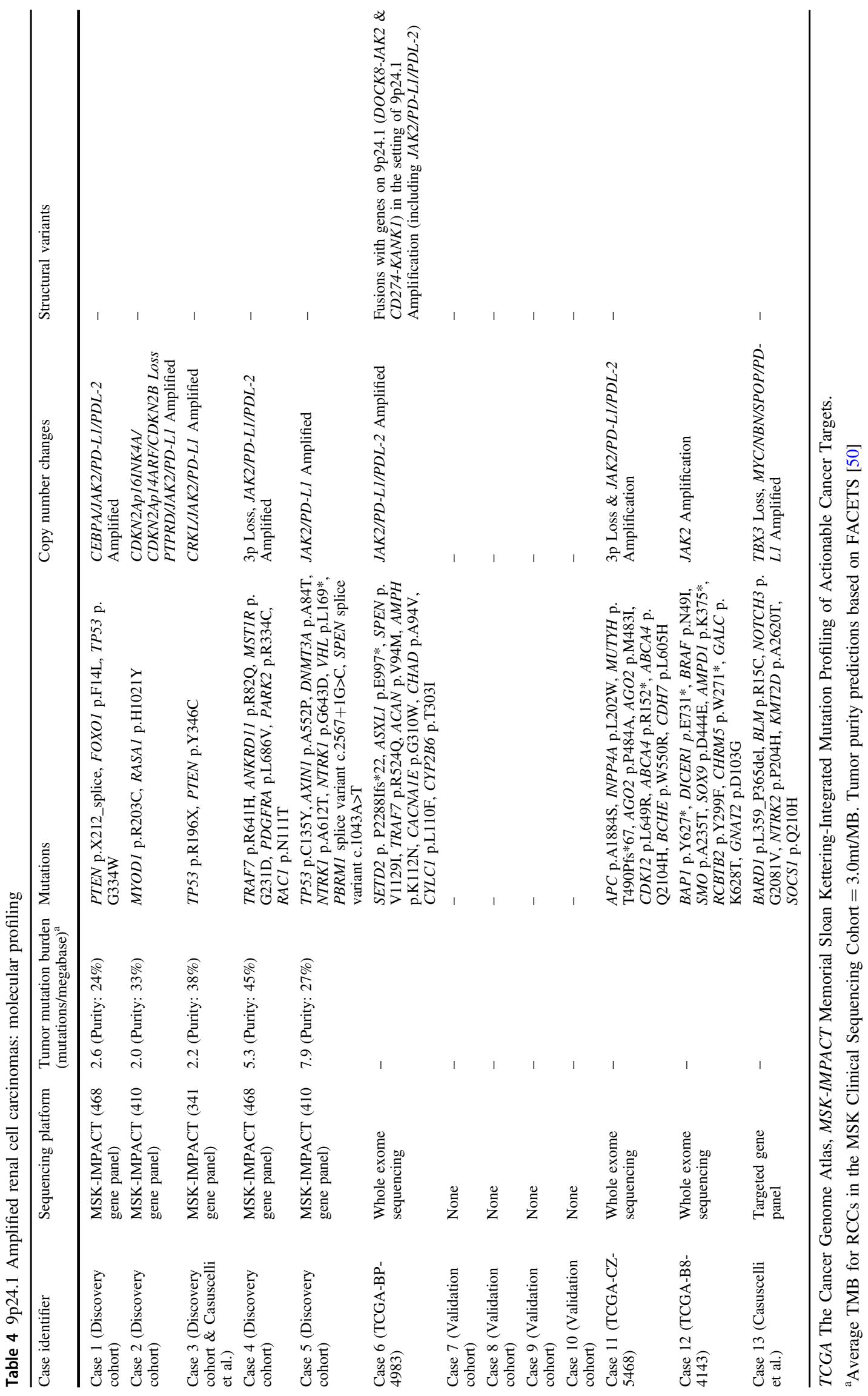


A

H-Score: PD-L1

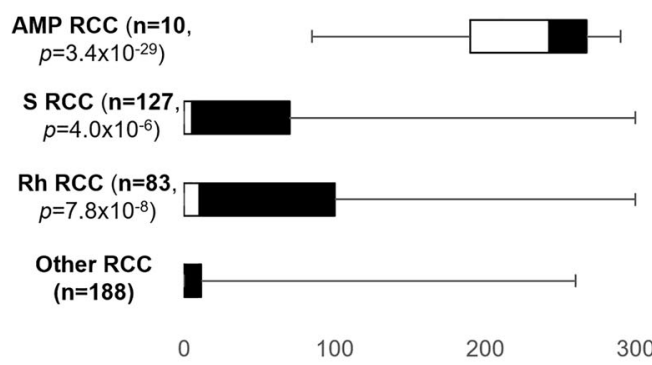

C

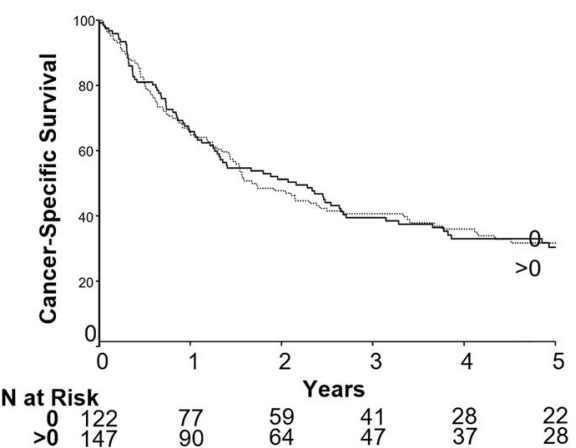

E

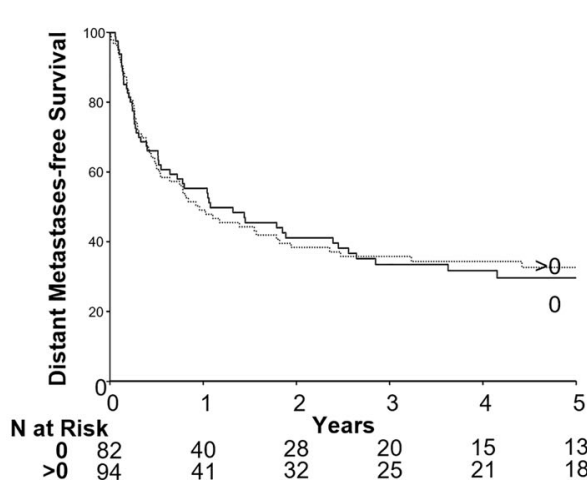

Fig. 4 PD-L1 expression status of high-grade renal cell carcinomas: prognostic significance. $H$-scores for PD-L1 expression based on immunohistochemistry was calculated semi quantitatively as the product of intensity of staining (graded from 0 to 3 ) and the percentage of positive cells showing membranous staining (0 to 100\%) for 398 renal cell carcinomas. The range of distribution of $\mathrm{H}$-Scores show significantly higher expression for 9p24.1 amplified renal tumors compared to cases without this genomic signature (a). The percentage of

L1 $H$-scores evaluated as continuous and categorical with cancer-specific survival is illustrated in Fig. $3 \mathrm{~d}$ and summarized in Supplementary Table 2. Distant metastases-free survival rates (95\% confidence interval; number still at risk) at 1,2 , and 3 years following surgery were $52 \%(45-60 ; 81)$, $40 \%(33-48 ; 60)$, and $35 \%(28-43 ; 45)$, respectively (Fig. 3e). The univariable association with distant metastasesfree survival among patients who were M0 at nephrectomy is illustrated in Fig. 3f and summarized in Supplementary Table 2 . There were no statistically significant interactions
B

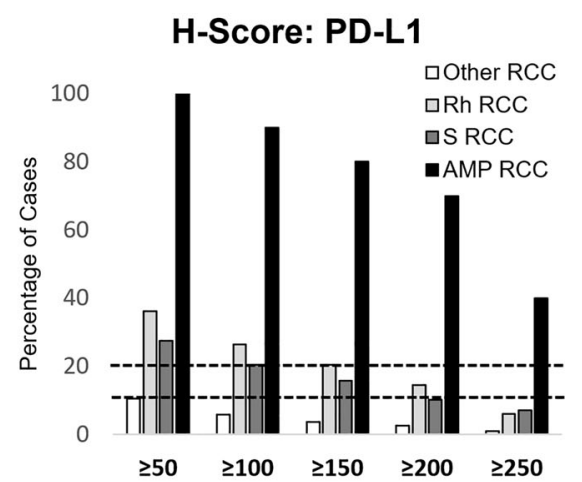

D

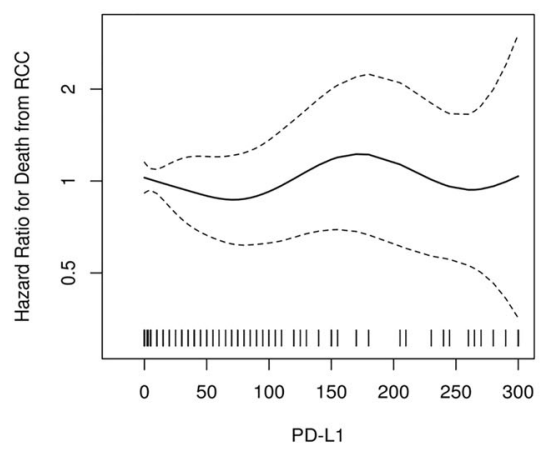

$\mathbf{F}$

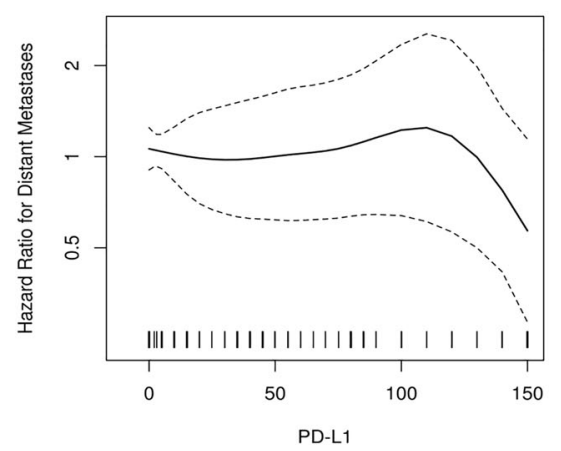

indicated sub-categories of renal tumors that have a $H$-Score $\geq 50$, $\geq 100, \geq 150, \geq 200$, and $\geq 250$ is shown (b). Univariable associations of categorized (c) and continuous (d) PD-L1 $H$-scores with cancerspecific survival are depicted. In addition, univariable associations of categorized (e) and continuous (f) PD-L1 H-scores with distant metastasis-free survival are depicted. S RCC: sarcomatoid renal cell carcinoma; Rh RCC: renal cell carcinoma with rhabdoid features; Amp RCC: 9p24.1 amplified renal cell carcinoma

between the categorized PD-L1 H-scores and rhabdoid ( $p=$ $0.45)$ or sarcomatoid $(p=0.62)$ differentiation for cancerspecific survival or distant metastasis-free survival (rhabdoid: $p=0.27$, sarcomatoid: $p=0.66$ ).

\section{Discussion}

This study was aimed at characterizing renal tumors with constitutive expression of JAK2/PD-L1 and $P D-L 2$, 
secondary to genomic amplification events. Herein we highlight a unique biologic phenomenon where a lung adenocarcinoma with an isolated germline intragenic gain of $J A K 2$ (including the entire kinase domain) shows increased phosphorylation of the JAK2 signaling target: STAT3. This in turn was correlated with overexpression of PD-L1 in all the neoplastic cells, consistent with activation of this signaling pathway. This supports the hypothesis that genomic amplifications at 9p24.1 upregulate PD-L1 expression both through the amplification of the $P D-L 1$ gene and secondary to JAK2 activation. Furthermore, our results are consistent with the findings of Zhao et al. in that other than 9p24.1 amplification, activating JAK2 alterations were not identified in any renal tumor subjected to molecular profiling, and if present at low variant allele fractions are likely to represent clonal hematopoiesis events [44, 45].

Next generation sequencing based assessment of copy number gains at the 9p24.1 locus does not account for tumor heterogeneity due to the presence of non-neoplastic elements such as tumor infiltrating lymphocytes, stromal elements and background renal parenchyma. Therefore, the intra-tumoral localization of this genomic signature was confirmed using a combination of fluorescence in situ hybridization and immunohistochemistry which revealed these alterations to be limited to the tumor cells. RNA sequencing data and immunohistochemistry confirmed downstream changes at the mRNA and protein level, respectively. Interestingly, the strongest correlation between 9p24.1 amplification status and immunohistochemistry was for PD-L1 expression. As mentioned earlier, this may be explained by the fact that, in addition to genomic amplification, $P D-L 1$ is also upregulated secondary to JAK2 activation. Furthermore, it is not uncommon to see $9 \mathrm{p} 24.1$ amplified tumors exhibit a combined pattern of PD-L1 expression, as several of these tumors exhibited constitutive expression along with adaptive expression that was geographically associated with and presumably induced by activated T-cell infiltrates [18]. Of note, a recent study suggested that renal tumors with PBRMI loss of function alterations exhibit increased activation of the JAK-STAT signaling pathway [46]. A single case showed a PBRMI splice site alteration (c.2567+1G $>C$, Case 5) and this could represent an additional mechanism of increased JAK2 signaling induced PD-L1 overexpression.

Our studies suggest that 9p24.1 amplifications are significantly enriched in renal cell carcinomas with sarcomatoid transformation $(5.95 \%$ versus $0.6 \%$ ). An interesting finding from cases identified in this cohort was the observation that in cases where a lower grade epithelial component was present, these alterations were only identified in the higher grade rhabdoid or sarcomatoid component. This observation is consistent with findings from a prior study where comprehensive molecular profiling was conducted both of a lower grade epithelial component and of a higher grade sarcomatoid component [38]. In 1 of 3 cases profiled in this manner, a 9p24.1 amplification was detected only in the sarcomatoid component [38]. Future studies are required to confirm this observation.

A review of the literature and our results suggests that 9p24.1 amplifications can occur in renal tumors with sarcomatoid transformation arising in a background of both clear cell and chromophobe renal cell carcinoma subtypes $[37,38]$. This raises the possibility that 9p24.1 amplifications may promote or be associated with sarcomatoid transformation independent of the underlying tumor type. At least one prior study has suggested that PD-L1 could directly govern sarcomatoid transformation by inducing epithelial to mesenchymal transformation in renal cell carcinomas $[47,48]$.

As prior studies have shown prognostic implications of PD-L1 expression status in renal tumors, including those with sarcomatoid transformation, we assessed PD-L1 expression in a cohort of 398 high-grade renal cell carcinomas [20, 22, 24, 25, 43]. Our observations indicate that higher grade elements within a tumor often exhibit higher PD-L1 expression. Considering this, our approach of scoring PD-L1 expression in tissue microarray specimens was limited by tumor heterogeneity. However, this approach mirrors actual clinical practice for other tumor types. For instance, PD-L1 expression status in limited lung biopsies is often used to guide therapeutic decision making [49]. No significant prognostic effects of PD-L1 expression was noted amongst our cohort of high-grade renal tumors on cancer-specific survival or distant metastasis-free survival and it must be noted that only a minor subset of these patients received immunotherapy. This likely reflects the advanced disease status of our patient cohort selected for statistical analysis and is highlighted by the fact that at last follow-up 229 (of 282) patients had died at a median of 1.3 years following surgery. Although PD-L1 expression status in this cohort did not have a significant prognostic impact, it is imperative to identify biomarkers of response to therapy within this group of patients.

Interestingly, 2 patients with 9p24.1 amplified sarcomatoid renal cell carcinomas in our study demonstrated promising response to immunotherapy. While this is a very limited number of patients, these results are encouraging and suggest that 9p24.1 amplifications in renal tumors may be a biomarker for exceptional response to immunotherapy. This is consistent with what has been reported for response to immunotherapy for other 9p24.1-amplified hematologic and solid tumors [3,12-17]. Our results suggest a need for future studies that correlate response to immunotherapy with constitutive PD-L1 expression in advanced renal tumors.

Currently, some of the alternate next generation sequencing-based metrics which can potentially be used to 
guide immunotherapeutic decision making include an assessment of tumor mutation burden but our results suggest that this may not be a useful biomarker in patients with 9p24.1 amplified renal tumors.

In summary, 9p24.1 amplifications are enriched in renal tumors with sarcomatoid transformation and the prevalence of this alteration is approximately $5.95 \%$ of all such cases. This genomic amplification event shows good concordance with PD-L1 immunohistochemistry and this can be utilized in routine clinical practice to screen for these events. As immune checkpoint inhibitors continue to gain popularity as a therapeutic modality for advanced renal cell carcinomas, our study helps identify potential patient populations who might exhibit enhanced response to these agents [19-25].

Acknowledgements This study was supported in part through NIH/ NCI Cancer Center Support grant P30CA008748.

\section{Compliance with ethical standards}

Conflict of interest The authors declare that they have no conflict of interest.

Publisher's note: Springer Nature remains neutral with regard to jurisdictional claims in published maps and institutional affiliations.

\section{References}

1. Shuai K, Liu B. Regulation of JAK-STAT signalling in the immune system. Nat Rev Immunol. 2003;3:900-11.

2. Levine RL, Pardanani A, Tefferi A, Gilliland DG. Role of JAK2 in the pathogenesis and therapy of myeloproliferative disorders. Nat Rev Cancer. 2007;7:673-83.

3. Goodman AM, Piccioni D, Kato S, Boichard A, Wang HY, Frampton G, et al. Prevalence of PDL1 amplification and preliminary response to immune checkpoint blockade in solid tumors. JAMA Oncol. 2018;4:1237-44

4. Green MR, Monti S, Rodig SJ, Juszczynski P, Currie T, O'Donnell E, et al. Integrative analysis reveals selective 9p24.1 amplification, increased PD-1 ligand expression, and further induction via JAK2 in nodular sclerosing Hodgkin lymphoma and primary mediastinal large B-cell lymphoma. Blood. 2010; 116:3268-77.

5. Cancer Genome Atlas Research Network. Comprehensive molecular characterization of gastric adenocarcinoma. Nature. 2014;513:202-9.

6. Straub M, Drecoll E, Pfarr N, Weichert W, Langer R, Hapfelmeier A, et al. CD274/PD-L1 gene amplification and PD-L1 protein expression are common events in squamous cell carcinoma of the oral cavity. Oncotarget. 2016;7:12024-34.

7. George J, Saito M, Tsuta K, Iwakawa R, Shiraishi K, Scheel AH, et al. Genomic amplification of CD274 (PD-L1) in small-cell lung cancer. Clin Cancer Res. 2017;23:1220-6.

8. Balko JM, Giltnane JM, Wang K, Schwarz LJ, Young CD, Cook RS, et al. Molecular profiling of the residual disease of triple-negative breast cancers after neoadjuvant chemotherapy identifies actionable therapeutic targets. Cancer Discov. 2014;4:232-45.

9. Barrett MT, Anderson KS, Lenkiewicz E, Andreozzi M, Cunliffe HE, Klassen CL, et al. Genomic amplification of 9p24.1 targeting
JAK2, PD-L1, and PD-L2 is enriched in high-risk triple negative breast cancer. Oncotarget. 2015;6:26483-93.

10. Balko JM, Schwarz LJ, Luo N, Estrada MV, Giltnane JM, DávilaGonzález D, et al. Triple-negative breast cancers with amplification of JAK2 at the 9p24 locus demonstrate JAK2-specific dependence. Sci Transl Med. 2016;8:334ra53.

11. Chen M, Andreozzi M, Pockaj B, Barrett MT, Ocal IT, McCullough AE, et al. Development and validation of a novel clinical fluorescence in situ hybridization assay to detect JAK2 and PD-L1 amplification: a fluorescence in situ hybridization assay for JAK2 and PD-L1 amplification. Mod Pathol. 2017;30:1516-26.

12. Gupta S, Vanderbilt CM, Cotzia P, Arias-Stella JA 3rd, Chang JC, Zehir A, et al. Next-generation sequencing-based assessment of JAK2, PD-L1, and PD-L2 copy number alterations at 9p24.1 in breast cancer: potential implications for clinical management. J Mol Diagn. 2018;21:307-17.

13. Ansell SM, Lesokhin AM, Borrello I, Halwani A, Scott EC, Gutierrez M, et al. PD-1 blockade with nivolumab in relapsed or refractory Hodgkin's lymphoma. N Engl J Med. 2015;372:311-9.

14. Nayak L, Iwamoto FM, LaCasce A, Mukundan S, Roemer MGM, Chapuy B, et al. PD-1 blockade with nivolumab in relapsed/ refractory primary central nervous system and testicular lymphoma. Blood. 2017;129:3071-3.

15. Younes A, Santoro A, Shipp M, Zinzani PL, Timmerman JM, Ansell S, et al. Nivolumab for classical Hodgkin's lymphoma after failure of both autologous stem-cell transplantation and brentuximab vedotin: a multicentre, multicohort, single-arm phase 2 trial. Lancet Oncol. 2016;17:1283-94.

16. Ikeda S, Goodman AM, Cohen PR, Jensen TJ, Ellison CK, Frampton G, et al. Metastatic basal cell carcinoma with amplification of PD-L1: exceptional response to anti-PD1 therapy. NPJ Genom Med 2016;1. https://doi.org/10.1038/npjgenmed.2016.37.

17. Gupta S, Vanderbilt CM, Cotzia P, Arias Stella JA 3rd, Chang JC, Chen Y, et al. JAK2, PD-L1, and PD-L2 (9p24.1) Amplification in Metastatic Mucosal and Cutaneous Melanomas with Durable Response to Immunotherapy. Hum Pathol. 2018. pii: S0046-8177(18)30360-5. https://doi.org/10.1016/j.humpath.2018. 08.032. [Epub ahead of print].

18. Taube JM, Galon J, Sholl LM, Rodig SJ, Cottrell TR, Giraldo NA, et al. Implications of the tumor immune microenvironment for staging and therapeutics. Mod Pathol. 2018;31:214-34.

19. Motzer RJ, Escudier B, McDermott DF, George S, Hammers HJ, Srinivas S, et al. Nivolumab versus everolimus in advanced renalcell carcinoma. N Engl J Med. 2015;373:1803-13.

20. Motzer RJ, Tannir NM, McDermott DF, Arén Frontera O, Melichar B, Choueiri TK, et al. Nivolumab plus ipilimumab versus sunitinib in advanced renal-cell carcinoma. $\mathrm{N}$ Engl $\mathrm{J}$ Med. 2018;378:1277-90.

21. Escudier B, Sharma P, McDermott DF, George S, Hammers HJ, Srinivas $\mathrm{S}$, et al. CheckMate 025 randomized phase 3 study: outcomes by key baseline factors and prior therapy for nivolumab versus everolimus in advanced renal cell carcinoma. Eur Urol. 2017;72:962-71.

22. Joseph RW, Millis SZ, Carballido EM, Bryant D, Gatalica Z, Reddy S, et al. PD-1 and PD-L1 expression in renal cell carcinoma with sarcomatoid differentiation. Cancer Immunol Res. 2015;3:1303-7.

23. McDermott DF, Sosman JA, Sznol M, Massard C, Gordon MS, Hamid $\mathrm{O}$, et al. Atezolizumab, an anti-programmed death-ligand 1 antibody, in metastatic renal cell carcinoma: long-term safety, clinical activity, and immune correlates from a phase Ia study. J Clin Oncol. 2016;34:833-42.

24. Thompson RH, Gillett MD, Cheville JC, Lohse CM, Dong H, Webster WS, et al. Costimulatory B7-H1 in renal cell carcinoma patients: Indicator of tumor aggressiveness and potential therapeutic target. Proc Natl Acad Sci USA. 2004;101:17174-9. 
25. Rini BI, Huseni M, Atkins MB, McDermott DF, Powles TB, Escudier B, et al. LBA31Molecular correlates differentiate response to atezolizumab (atezo)+bevacizumab (bev) vs sunitinib (sun): Results from a phase III study (IMmotion151) in untreated metastatic renal cell carcinoma (mRCC). Ann Oncol. 2018;29, https://doi.org/10.1093/annonc/mdy424.037.

26. Cheng DT, Mitchell TN, Zehir A, Shah RH, Benayed R, Syed A, et al. Memorial sloan kettering-integrated mutation profiling of actionable cancer targets (MSK-IMPACT): a hybridization capture-based next-generation sequencing clinical assay for solid tumor molecular oncology. J Mol Diagn. 2015;17:251-64.

27. Hyman DM, Solit DB, Arcila ME, Cheng DT, Sabbatini P, Baselga J, et al. Precision medicine at Memorial Sloan Kettering Cancer Center: clinical next-generation sequencing enabling nextgeneration targeted therapy trials. Drug Discov Today. 2015;20:1422-8.

28. Zehir A, Benayed R, Shah RH, Syed A, Middha S, Kim HR, et al. Mutational landscape of metastatic cancer revealed from prospective clinical sequencing of 10,000 patients. Nat Med. 2017;23:703-13.

29. Italiano A, Thomas R, Breen M, Zhang L, Crago AM, Singer S, et al. The miR-17-92 cluster and its target THBS1 are differentially expressed in angiosarcomas dependent on MYC amplification. Genes Chromosomes Cancer. 2012;51:569-78.

30. Roemer MG, Advani RH, Ligon AH, Natkunam Y, Redd RA, Homer $\mathrm{H}$, et al. PD-L1 and PD-L2 genetic alterations define classical Hodgkin lymphoma and predict outcome. J Clin Oncol. 2016;34:2690-7.

31. Ross DS, Zehir A, Cheng DT, Benayed R, Nafa K, Hechtman $\mathrm{JF}$, et al. Next-generation assessment of human epidermal growth factor receptor 2 (ERBB2) amplification status: clinical validation in the context of a hybrid capture-based, comprehensive solid tumor genomic profiling assay. J Mol Diagn. 2017; 19:244-54.

32. Gao J, Aksoy BA, Dogrusoz U, Dresdner G, Gross B, Sumer SO, et al. Integrative analysis of complex cancer genomics and clinical profiles using the cBioPortal. Sci Signal. 2013;6:pl1.

33. Cancer Genome Atlas Research Network. Comprehensive molecular characterization of clear cell renal cell carcinoma. Nature. 2013;499:43-9.

34. Cancer Genome Atlas Research Network, Linehan WM, Spellman PT, Ricketts CJ, Creighton CJ, Fei SS, et al. Comprehensive molecular characterization of papillary renal-cell carcinoma. $\mathrm{N}$ Engl J Med. 2016;374:135-45.

35. Davis CF, Ricketts CJ, Wang M, Yang L, Cherniack AD, Shen H, et al. The somatic genomic landscape of chromophobe renal cell carcinoma. Cancer Cell. 2014;26:319-30.

36. Ricketts CJ, De Cubas AA, Fan H, Smith CC, Lang M, Reznik E, et al. The Cancer Genome Atlas comprehensive molecular characterization of renal cell carcinoma. Cell Rep. 2018;23:3698.
37. Casuscelli J, Weinhold N, Gundem G, Wang L, Zabor EC, Drill E, et al. Genomic landscape and evolution of metastatic chromophobe renal cell carcinoma. JCI Insight 2017;2.

38. Malouf GG, Ali SM, Wang K, Balasubramanian S, Ross JS, Miller VA, et al. Genomic characterization of renal cell carcinoma with sarcomatoid dedifferentiation pinpoints recurrent genomic alterations. Eur Urol. 2016;70:348-57.

39. Kalyana-Sundaram S, Shankar S, Deroo S, Iyer MK, Palanisamy $\mathrm{N}$, Chinnaiyan AM, et al. Gene fusions associated with recurrent amplicons represent a class of passenger aberrations in breast cancer. Neoplasia. 2012;14:702-8.

40. Gupta S, Johnson SH, Vasmatzis G, Porath B, Rustin JG, Rao P, et al. TFEB-VEGFA (6p21.1) co-amplified renal cell carcinoma: a distinct entity with potential implications for clinical management. Mod Pathol. 2017;30:998-1012.

41. Wang Z, Kim TB, Peng B, Karam J, Creighton C, Joon A, et al. Sarcomatoid renal cell carcinoma has a distinct molecular pathogenesis, driver mutation profile, and transcriptional landscape. Clin Cancer Res. 2017;23:6686-96.

42. Goodman AM, Kato S, Bazhenova L, Patel SP, Frampton GM, Miller V, et al. Tumor mutational burden as an independent predictor of response to immunotherapy in diverse cancers. Mol Cancer Ther. 2017;16:2598-608.

43. Kawakami F, Sircar K, Rodriguez-Canales J, Fellman BM, Urbauer DL, Tamboli P, et al. Programmed cell death ligand 1 and tumor-infiltrating lymphocyte status in patients with renal cell carcinoma and sarcomatoid dedifferentiation. Cancer. 2017;123:4823-31.

44. Zhao J, Moch H. Absence of JH2 domain mutation of the tyrosine kinase JAK2 in renal cell carcinomas. Acta Oncol. 2008;47:474-6.

45. Ptashkin RN, Mandelker DL, Coombs CC, Bolton K, Yelskaya Z, Hyman DM, et al. Prevalence of clonal hematopoiesis mutations in tumor-only clinical genomic profiling of solid tumors. JAMA Oncol. 2018;4:1589-93.

46. Miao D, Margolis CA, Gao W, Voss MH, Li W, Martini DJ, et al. Genomic correlates of response to immune checkpoint therapies in clear cell renal cell carcinoma. Science. 2018;359:801-6.

47. He H, Magi-Galluzzi C. Epithelial-to-mesenchymal transition in renal neoplasms. Adv Anat Pathol. 2014;21:174-80.

48. Wang Y, Wang H, Zhao Q, Xia Y, Hu X, Guo J. PD-L1 induces epithelial-to-mesenchymal transition via activating SREBP-1c in renal cell carcinoma. Med Oncol. 2015;32:212.

49. Forde PM, Chaft JE, Smith KN, Anagnostou V, Cottrell TR, Hellmann MD, et al. Neoadjuvant PD-1 blockade in resectable lung cancer. N Engl J Med. 2018;378:1976-86.

50. Shen R, Seshan VE. FACETS: allele-specific copy number and clonal heterogeneity analysis tool for high-throughput DNA sequencing. Nucleic Acids Res. 2016;44:e131 